Document downloaded from:

http://hdl.handle.net/10251/94473

This paper must be cited as:

Bloem, C.; Salvador Moya, MD.; Amigó, V.; Vicente-Escuder, A. (2000). Microstructural and strength study of MIG welded joints of AW7020 aluminium alloy, as a function of joint geometry. Welding International. 14(12):970-974. doi:10.1080/09507110009549300

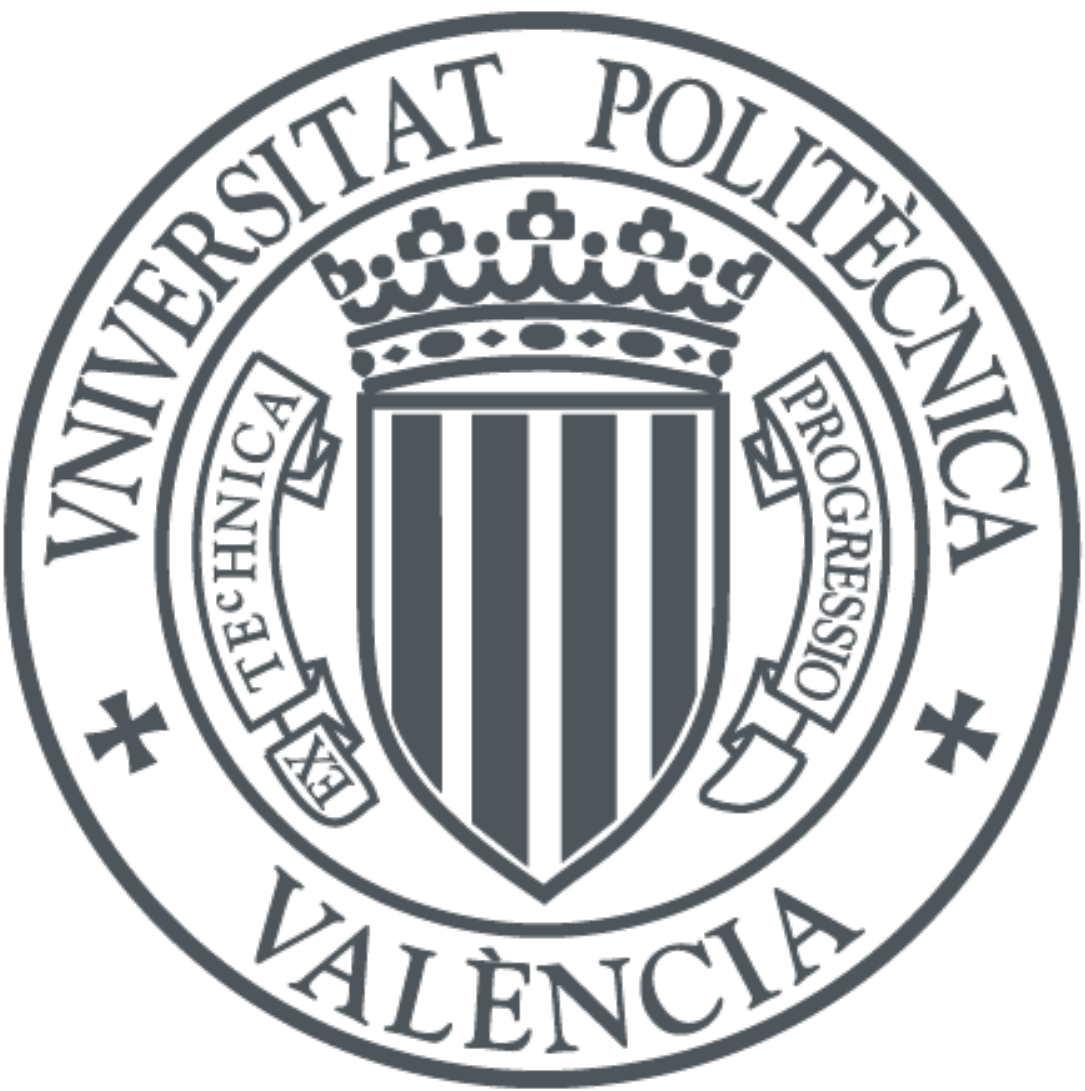

The final publication is available at

https://doi.org/10.1080/09507110009549300

Copyright Taylor \& Francis

Additional Information 


\section{Microstructural and strength study of MIG welded joints of AW7020 aluminium alloy, as a function of joint geometry}

\section{C.A. BLOEM}

Faculty of Engineering, School of Mechanical Engineering, University of the Andes, Mérida

M.D. SALVADOR, V. AMIGÓ and A. VICENTE

Department of Mechanical Engineering and Materials, Universidad Politécnica de Valencia

Summary: Medium strength AW7XXX aluminium alloys are widely used as welded structures and in transportation. The applications of these alloys are limited by the behaviour of the welded joints. There is no agreement on the joint geometry that must be used on $5 \mathrm{~mm}$ welds. The microhardness evolution is one of the most important strength indicators. For this reason, the aim of this work is to study the influence of welded joint geometry on microhardness profile and on the microstructure of a MIG welded AW7020 aluminium alloy, using AW5256 filler.

\section{Introduction}

Increasing use is made of aluminium and its alloys, which is mainly due to its low weight/strength ratio, and its high resistance to corrosion and precipitation hardening, discovered by accident at the beginning of the century by Alfred Wilm. ${ }^{1}$

The use of aluminium was restricted to small pieces or pieces not requiring welding, as aluminium could not be welded satisfactorily. Consequently, numerous articles were written on the subject of welding aluminium alloys. Many researchers have studied in depth the mechanical properties of the welded joint ${ }^{2}$ or were dedicated to metallurgical phenomena. ${ }^{3-5}$ Some have made comparisons between different welding methods, ${ }^{6-7}$ while others have studied post-weld treatment, ${ }^{8}$ but very few investigations were made of pre- and post-weld mechanical preparations. In addition, the heat input inherent to the welding process substantially affects the microstructure and its properties in the vicinity of the weld, i.e. the heat affected zone (HAZ). For this reason, the objective of this work is to qualify, by evaluating the microhardness in the HAZ, the efficiency of edge preparation before welding.

The aluminium welding process most widely accepted in industry is GMAW (MIG) due to its excellent quality, low cost and the lack of flux and/or solid protection which may be detrimental to welded joint quality. ${ }^{7}$

Of the heat-treatable aluminium alloys, those in series 7XXX with a total $\mathrm{Zn}+\mathrm{Mg}+\mathrm{Cu}$ content of alloying elements exceeding $9 \%$ have low weldability, but if this content is below $6 \%$, weldability is high. ${ }^{9}$

Due to its high weldability, alloys such as AW7005, AW7020 and AW7039 are widely used in welded structures. Nevertheless, the ASM 'Specialty handbook. Aluminium and aluminium alloys ${ }^{10}$ recommends the use of edge preparation from $5 \mathrm{~mm}$, while other sources ${ }^{10}$ recommend this from $8 \mathrm{~mm}$. Some researchers ${ }^{11}$ carry out edge preparation on plates with thicknesses much lower than those above. For this reason, it is necessary to study the effect of edge preparation on plates $5 \mathrm{~mm}$ thick or below, as well as its effect on the HAZ of series 7XXX aluminium alloys, which was not studied in depth. 
Selection of this alloy is influenced by its applicability to land transport and being a high productivity sector, it is perhaps one of the most resistant to applying this light alloy to its designs, which is understandable due to the myth that 'aluminium cannot be welded'. Therefore, considering the quality required in this sector, it was decided to carry out a study of medium strength AW7020 alloy which, among other characteristics, has acceptable weldability and accelerated natural ageing.

\section{Experimental procedure}

Materials used

The base metal for investigation came from a rolled AW7020 T6 plate supplied by Alumafel, with a chemical composition as shown in Table 1.

Different sources ${ }^{10,11}$ recommend the Al-Mg alloy known as AW5356 as filler metal, in the form of $1.2 \mathrm{~mm}$ diameter wire, whose chemical composition is also shown in Table 1.

Table 1 Chemical composition, mass \%

\begin{tabular}{|c|c|c|c|c|c|c|}
\hline & $\mathrm{Mg}$ & $\mathrm{Si}$ & $\mathrm{Cr}$ & $\mathrm{Mn}$ & $\mathrm{Zn}$ & $\mathrm{Fe}$ \\
\hline $\begin{array}{c}\text { Base metal } \\
\text { AW7020 }\end{array}$ & 1.2 & 0.17 & 0.31 & 0.20 & 5.05 & 0.25 \\
\hline Filler AW5356 & 5.0 & - & 0.12 & 0.15 & 0.1 & - \\
\hline
\end{tabular}

Welding

Welded joints were performed on four plates with dimensions of $350 \times 150 \times 5 \mathrm{~mm}$, two of which were welded with $+30^{\circ} \mathrm{V}$-bevel preparation while the others were butt welded, both being welded in two passes perpendicular to rolling direction; with mechanical cleaning using an abrasive disc between the first and second passes. The MIG-DC welding process was used with a 75\% Ar and 25\% He mixture as the shielding gas. The welding parameters are shown in Table 2.

Table 2 Welding parameters

\begin{tabular}{|l|l|l|l|l|l|}
\hline Condition & Angle & V, V & I, A & $\begin{array}{l}\text { Speed, } \\
\mathrm{mm} / \mathrm{min}\end{array}$ & EBA, kJ/cm \\
\hline $\begin{array}{l}\text { Without } \\
\text { bevel }\end{array}$ & - & 21.0 & 140 & $\begin{array}{l}525^{*} \\
382^{* *}\end{array}$ & $\begin{array}{l}5.6 \\
7.7\end{array}$ \\
\hline With bevel & $\pm 30^{\circ}$ & 19.3 & 120 & $\begin{array}{l}412^{*} \\
350^{* *}\end{array}$ & $\begin{array}{l}5.6 \\
6.6\end{array}$ \\
\hline
\end{tabular}

*First pass. ${ }^{* *}$ Second pass

Edge preparation of testpieces welded with V-bevel preparation was carried out using a grinding wheel, giving an angle of $30^{\circ}$, just before welding. Weld joints were cleaned by light brushing with a stainless steel bristle disc, maintaining joint morphology. Heel separation was $0.5 \mathrm{~mm}$ for joints with V-bevels and $1 \mathrm{~mm}$ for butt welded joints. In order to maintain distance and keep the sheets parallel during the welding process, they were adjusted with a pair of supports. 
Excess beading was completely removed using a grinding wheel to minimise any effect which could be caused by a difference in bead size.

Weld quality was assessed firstly by visual inspection, for lack of penetration or flaking. Welds were then examined using penetrating liquids before and after the removal of excess beading and were finally X-rayed, discarding those testpieces with linear defects or pores exceeding $0.5 \mathrm{~mm}$ in length or $1.0 \mathrm{~mm}$ in diameter.

Metallographic examination

Testpieces were prepared for metallographic examination using an optical microscope $(\mathrm{OM})$ and a scanning electron microscope (SEM) in order to evaluate possible microstructural differences between the two welding conditions, with or without bevel. In the same way, a quantitative analysis was carried out by X-ray microscopy. The equipment used was a Nikon Microphot FX model microscope and a Jeol model JSM63OO SEM, which was coupled to energy dispersive analysis by X-rays (EDAX) equipment.

\section{Mechanical properties}

Various microhardness scans were made along the transverse section of welded testpieces on the bead and along the HAZ according to standard ASTM E-384-73, ${ }^{13}$ using a Matsuzawa model MHT2 microhardness meter, with a Vickers-type indenter applying a load of 100 for 15 sec.

The microhardness spacing schedule followed by microhardness scans is similar to that used by Malin, ${ }^{14}$ with the variant evaluated every $0.1,0.3$ and $0.6 \mathrm{~mm}$ as seen in Fig. 1, where zero or the starting point is the interface between the bead (molten pool) and the base metal. The negative end is in the middle zone of the bead and the positive is at a distance of $32 \mathrm{~mm}$.

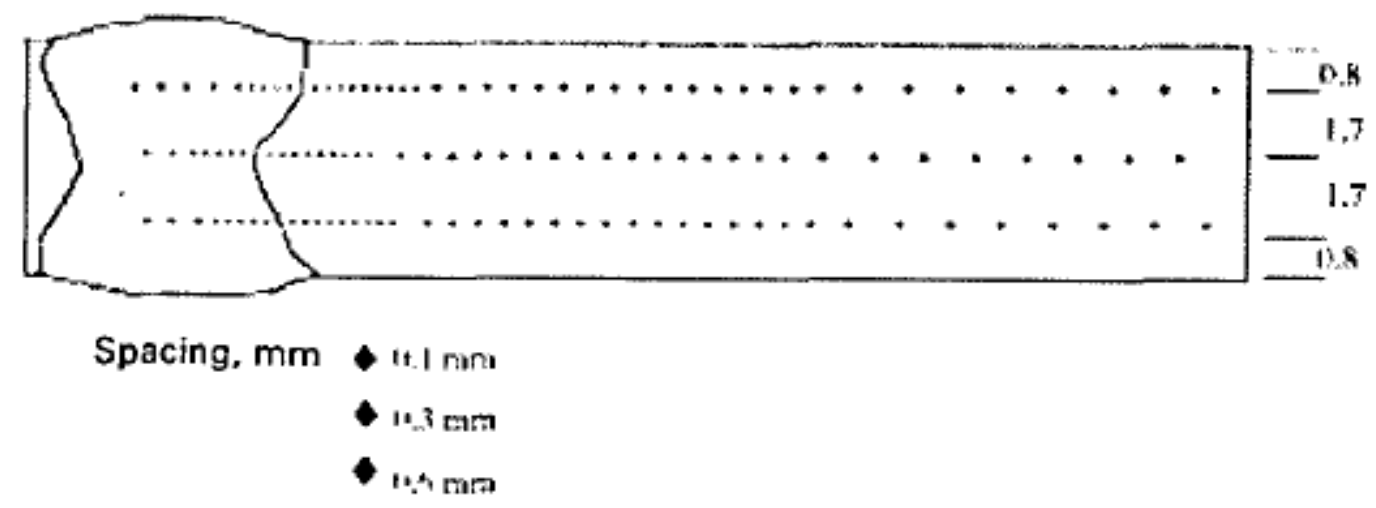

Fig 1 Microhardness spacing schedule.

In order to confirm microhardness results, axial tests were conducted according to standard UNE 7-474-92, EN 10-002-1, at a speed of $5 \mathrm{~mm} / \mathrm{min}$ on an Instron 4204 universal testing machine, with the average value of data obtained shown in Table 3.

Table 3 Axial test results

\begin{tabular}{|l|l|l|l|}
\hline Testpiece & $\begin{array}{l}\text { Elastic limit 0.2\%, } \\
\mathrm{MPa}\end{array}$ & $\begin{array}{l}\text { Ultimate stress, } \\
\mathrm{MPa}\end{array}$ & Elongation, \% \\
\hline Base metal & $344.4(100 \%)$ & $392.2(100 \%)$ & $15.37(100 \%)$ \\
\hline
\end{tabular}




\begin{tabular}{|l|l|l|l|}
\hline $\begin{array}{l}\text { Testpieces without } \\
\text { bevel }\end{array}$ & $195.1(57 \%)$ & $271.6(69 \%)$ & $6.07(40 \%)$ \\
\hline Bevelled testpieces & $229.0(66 \%)$ & $265.9(67 \%)$ & $5.69(37 \%)$ \\
\hline
\end{tabular}

Table 4 Welded zone dimensions

\begin{tabular}{|l|l|l|l|}
\hline Joint geometry & Area, $\mathrm{mm}^{2}$ & $\begin{array}{l}\text { Weld } \\
\text { reinforcement area }\end{array}$ & $\begin{array}{l}\text { Maximum and } \\
\text { minimum } \\
\text { distances, mm }\end{array}$ \\
\hline Without bevel & $34.22 \pm 9.8$ & 26.74 & $\begin{array}{l}9.39 \pm 2.1 \\
4.18+1.8\end{array}$ \\
\hline Bevel $+30^{\circ}$ & $31.21 \pm 5.9$ & 30.91 & $\begin{array}{l}8.93 \pm 1.6 \\
5.43 \pm 1.2\end{array}$ \\
\hline
\end{tabular}

\section{Results and discussion}

In order to evaluate the variation in bead size and dimensions solely in terms of the bevel, the average size of beads was measured using image digitisation of the transverse section of testpieces. The dimensions are shown in Table 4 and the macroscopic view can be seen in Fig. 2. From the above it can be observed that the average bead size of a bevelled weld is slightly greater than that of a weld with no bevel, measured over the largest area of the bead, being directly related with the heat supply as shown in Tables 2 and 4. Similarly, dispersion of bead dimensions with bevels is considerably lower than those without bevel, from which it may be inferred that repeatability of results is greater in joints with bevels. Nevertheless, it is evident that bead morphology of joints without beveled edges is more symmetrical in their transverse section. 


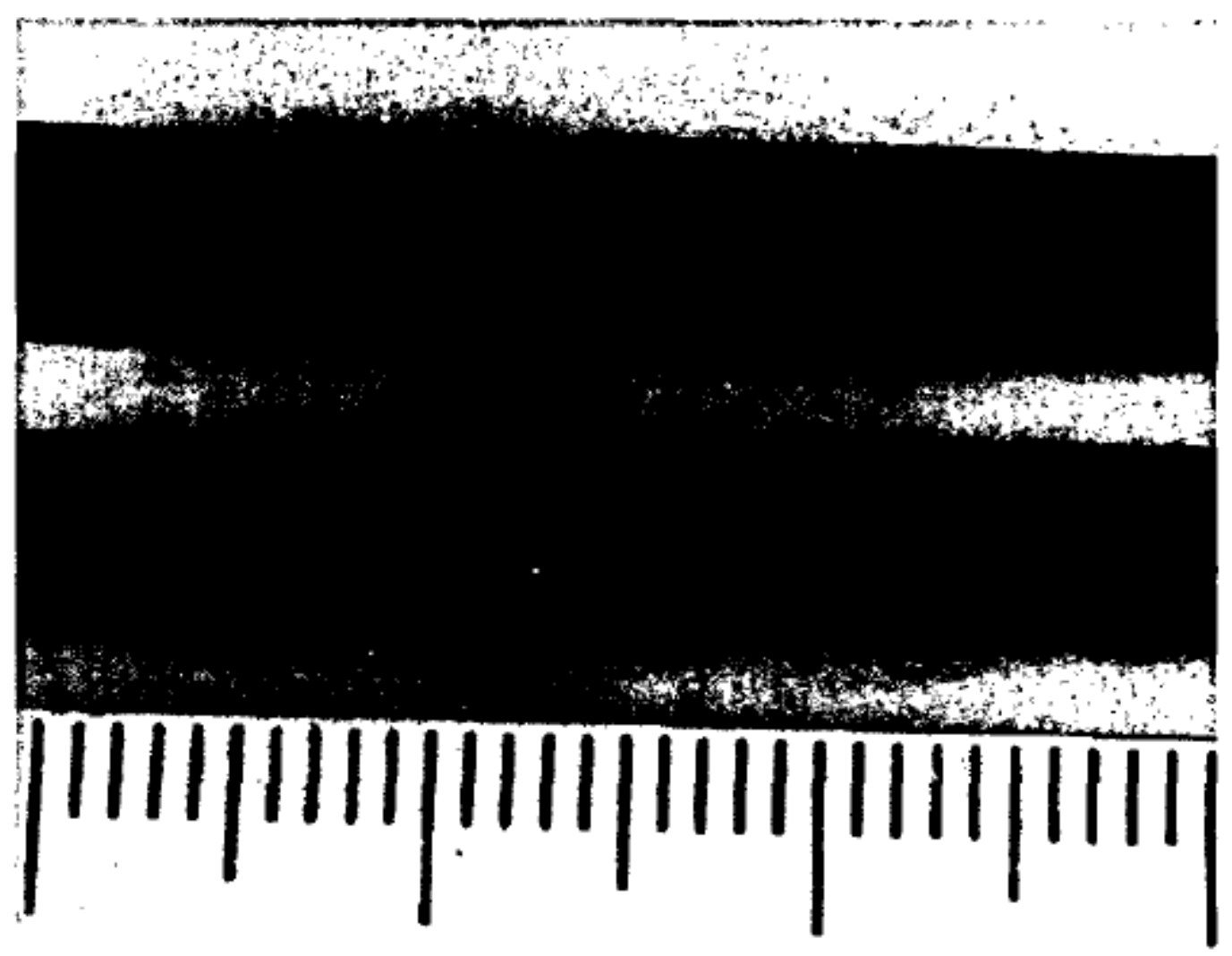

Fig. 2 Weld zone macrograph. Upper sample without V-bevel; lower one with $\pm 30^{\circ} \mathrm{V}$ bevel. Week's reagent.

The results of micro-analysis by EDAX equipment along the HAZ show that there is no volatility of alloying elements as shown by other researchers, 7 proving the appearance in all testpieces studied of relatively stable quantities of zinc, at around $2.2-3 \%$ along the bead (Fig. 3). This leads to the deduction that during the welding process, fusion of the base metal inside the bead is proportional to the quantity of filler metal added, joint morphology being of little importance. Nevertheless, it is observed that in bevelled testpieces, the zinc content is approximately 0.3 points higher, implying a $10 \%$ increase. The various analyses show that a new alloy in the Al-Zn-Mg family is formed in the bead, poorer in zinc but richer in magnesium (around 3\% Zn and slightly below 3\% $\mathrm{Mg}$ ), which agrees with the investigations by Yong-Seog ${ }^{8}$ and Hussain ${ }^{15}$, this being corroborated due to the presence of type $\mathrm{T}$ precipitates $(\mathrm{Al}, \mathrm{Zn})_{49} \mathrm{Mg}_{32}$. 


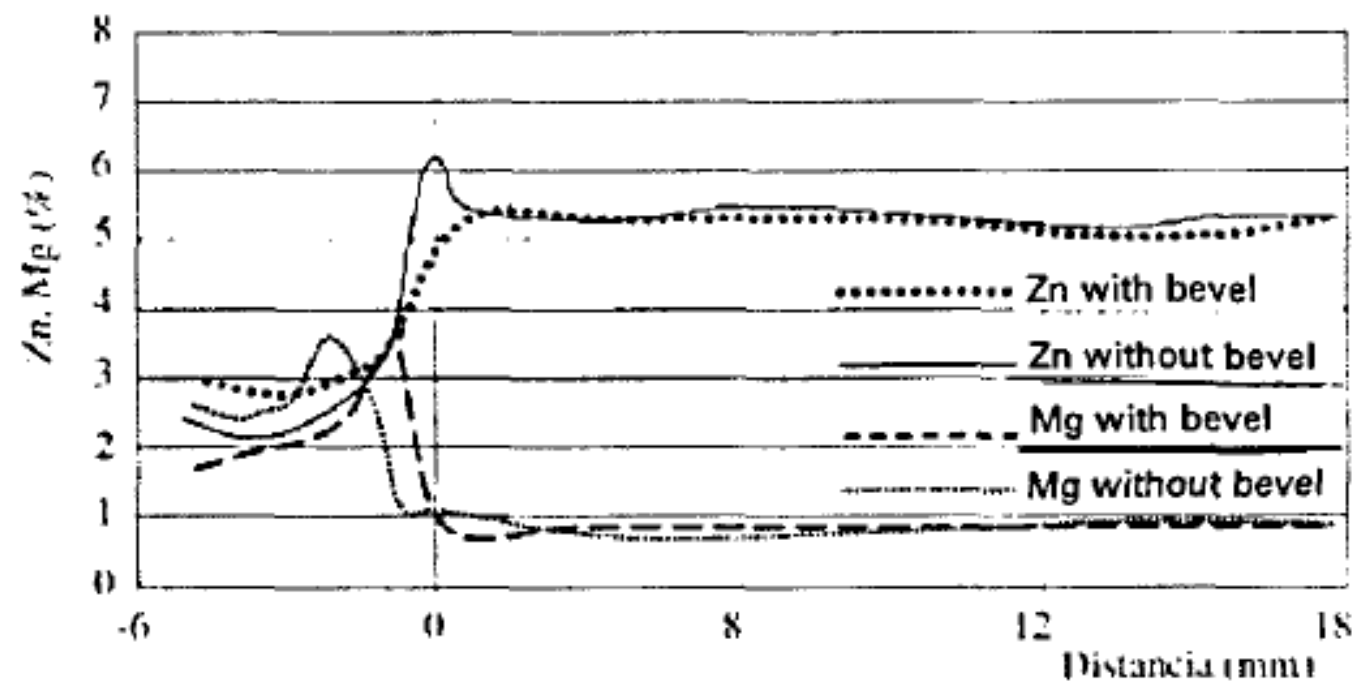

Fig. 3 Zinc and magnesium composition profile.

Nevertheless, microstructural evaluation of the two processes investigated does not show any appreciable difference between them, despite the fact that use of an OM with Week's reagent seems to show a slightly larger HAZ in testpieces with no bevel, which is not unusual due to a slightly increased EBA in welds with bevels.

As may be observed in Fig. 4, there are small pores in the bead which, given its almost spherical morphology, must be the product of hydrogen released during solidification. ${ }^{10-}$ 16

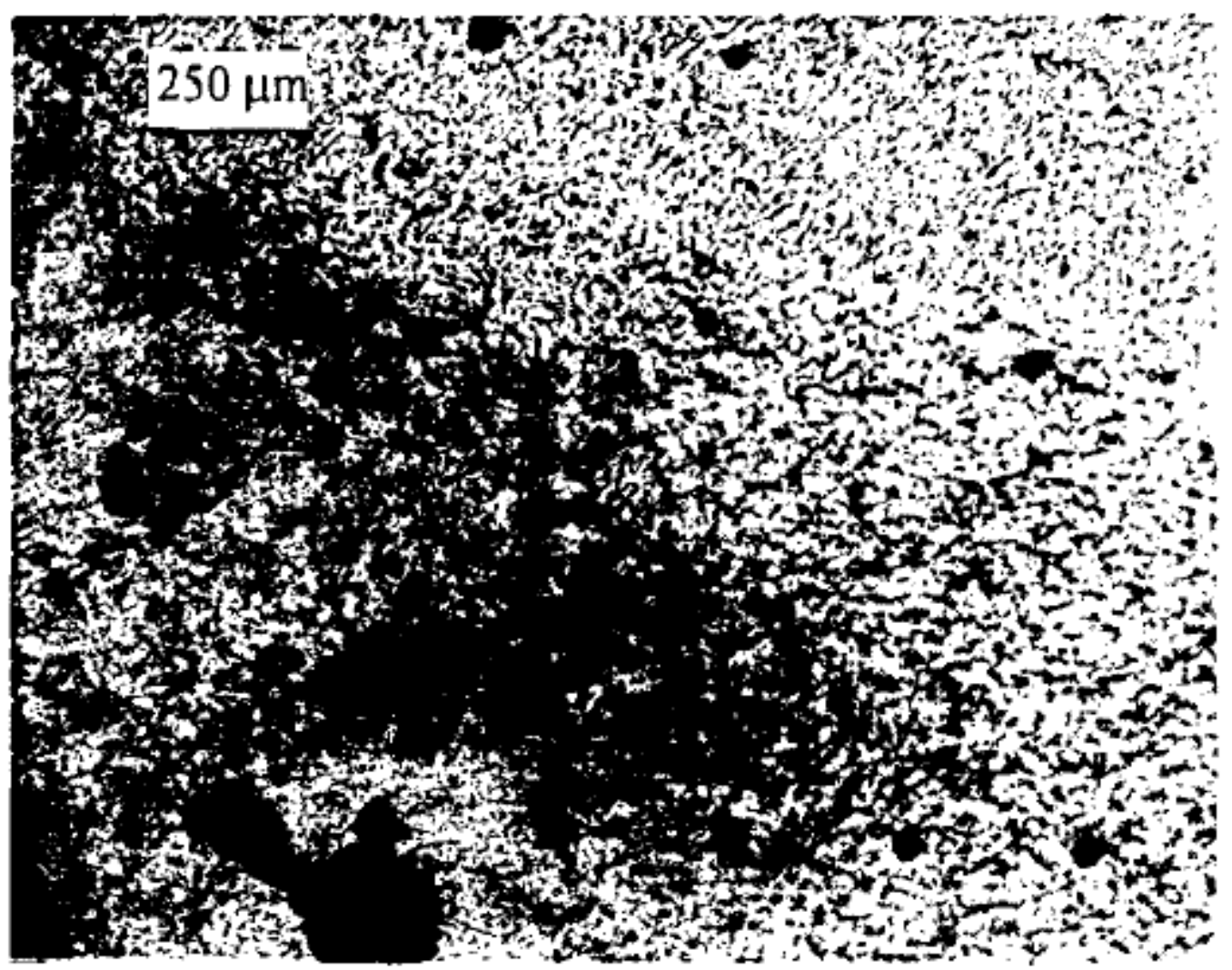

Fig 4 Micorstructure of base metal-welded zone interface. Keller's reagent, x50. 
Microstructural examination by an OM shows four different zones along the interface. The first with dendritic, columnar type grains, then an equiaxial zone with coarse grains, followed by another equiaxial zone with finer grains and, finally, the base metal.

In the coarse equiaxial grain zone, higher definition of grain boundaries was observed, this zone being known as a white zone by some authors. ${ }^{3}$ Its size varies between 0.5 and $1 \mathrm{~mm}$ and occurs only at the base metal-bead interface of series 7XXX alloy welds, as shown in Fig. 5.

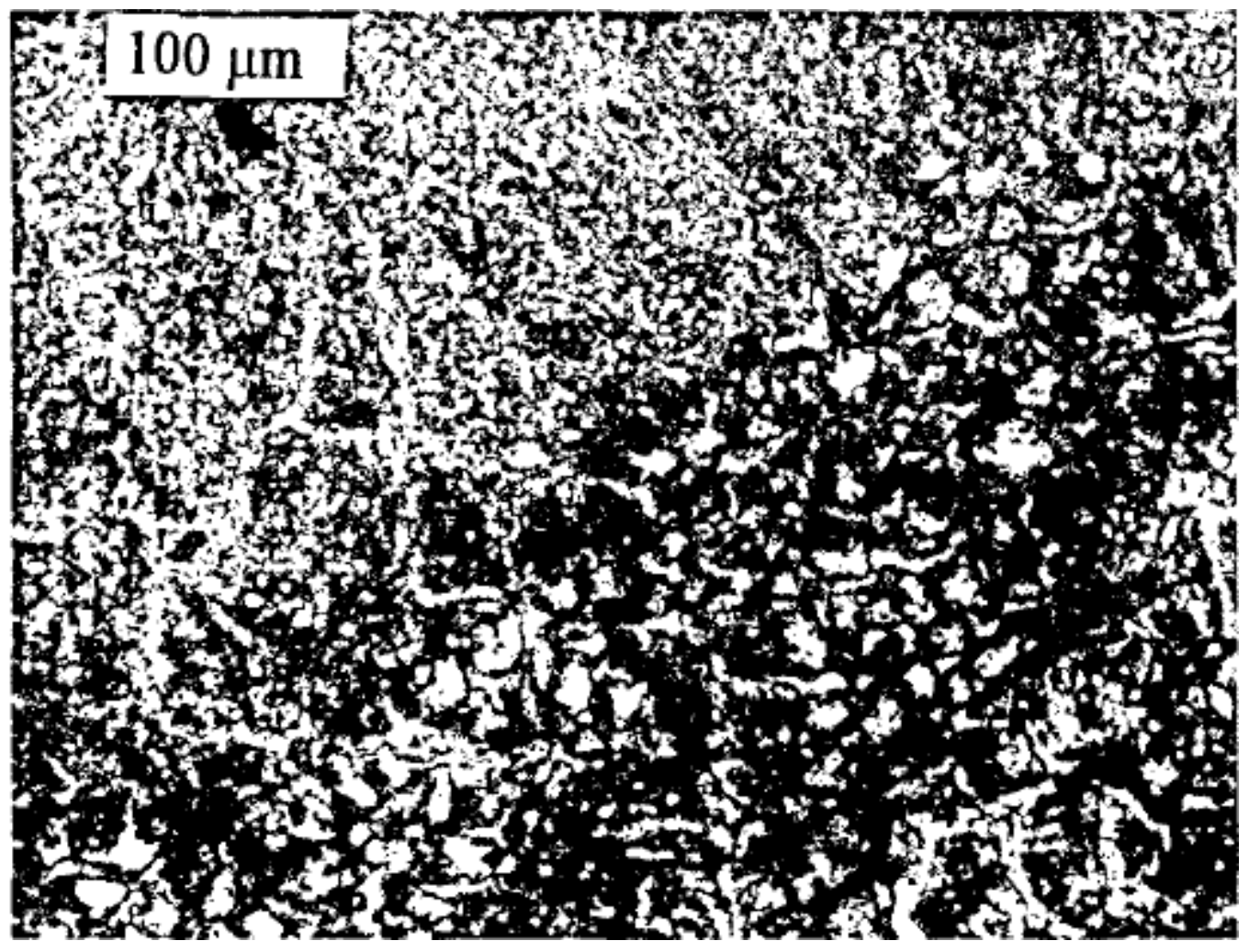

Fig. 5 White zone detail in welded zone-base metal interface. Week's reagent, x50.

Three microhardness scans were made (Fig. 6(a)) corroborating maximum or minimum values with new measurements in turn at these points. Figure 6(b) shows the resulting profiles in the different zones of a welded joint with no bevel. However, Fig. 7 shows profiles representing microhardness evolution along the welded joint with and without edge preparation, from the molten pool to the base metal. 

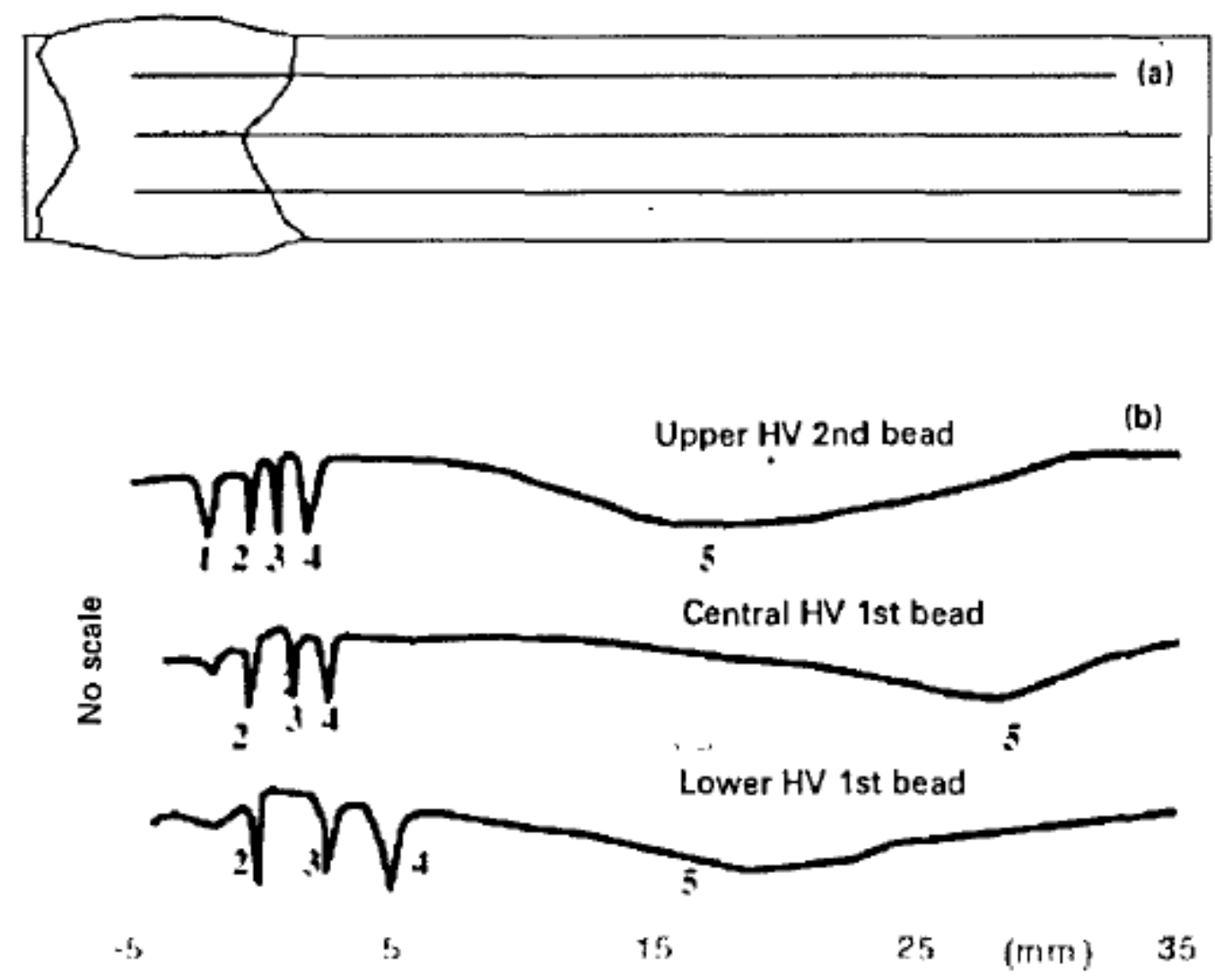

Fig. 6 Microhardness profile: a) Graph of microhardness measurement; $b$ ) Microhardness evolution of joint without V-bevel preparation.

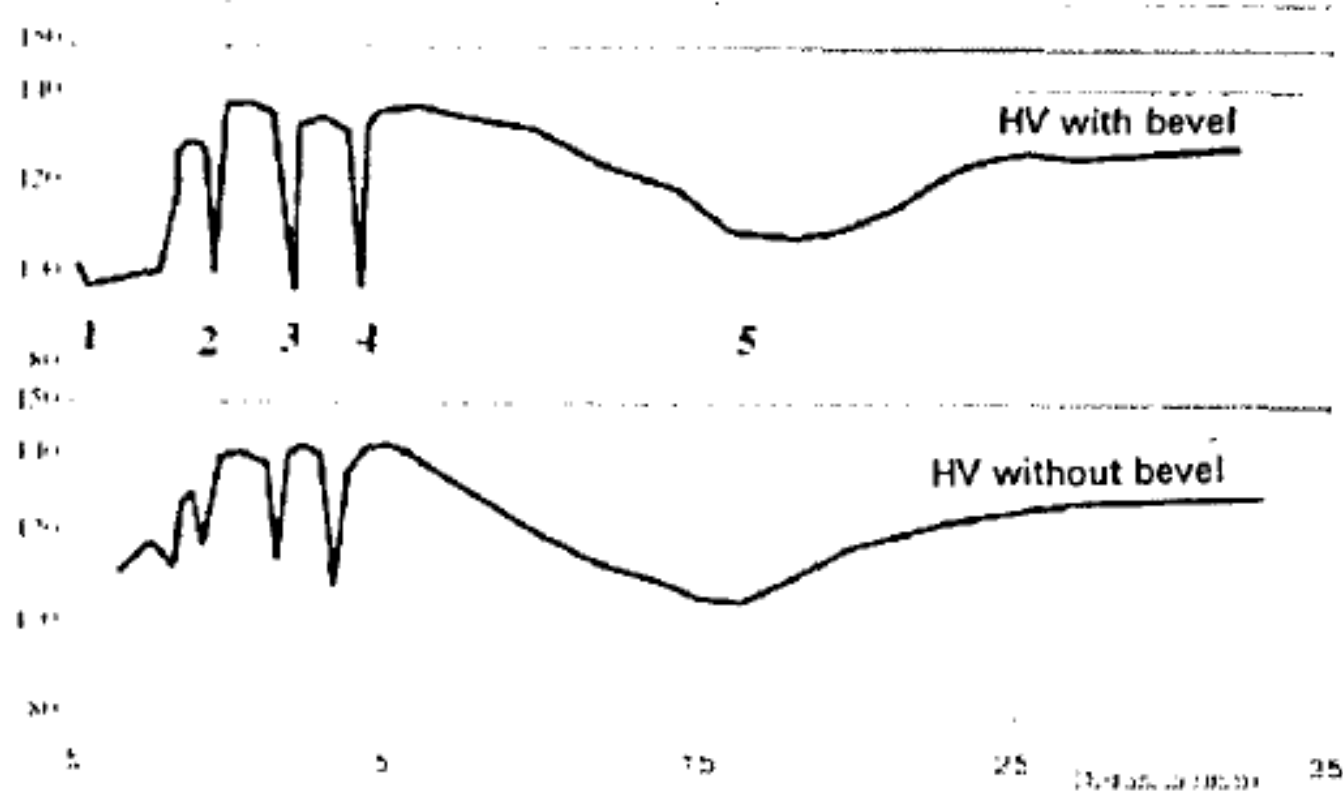

Fig 7 Upper side (2nd pass) microhardness evolution.

As may be observed in Fig. 6(b) and 7, five possible sites of mechanical failure may be determined, two within the bead and three in the HAZ. In the bead, the first site is situated approximately at the centre with a decrease in hardness of around 60-65\% compared with the base metal. This zone usually disappears into the bead corresponding to the first pass, which may be explained due to the great heat effect caused by the 
second pass on the material deposited in the first pass, generating dissolution, homogenisation and even precipitation of elements in the bead, as indicated by YongSeog8 based on the fact that the mixture of the base metal and the filler produces a new Al-Zn-Mg-Mn type alloy, as shown by the various microscopic analyses (EDAX) (Fig. $3)$.

The second weak zone occurs in a small region which may oscillate between $0.2-1 \mathrm{~mm}$ within the bead, with a gradual hardness decrease, to reach a minimum which in some cases may be up to $66 \%$ of base metal hardness value and may be justified by the residual stresses existing between the interface of fine and dendritic crystals.

The HAZ shows a third zone of decreased hardness with values which in some cases may be of the order of $68 \%$ of hardness value in the base metal, which is $0.5-3 \mathrm{~mm}$ from the fusion line. This zone is further away as heat input increases during the welding process.

The fourth zone corresponds to a large decrease in microhardness with the same features as the previous zone and may be confused with it or, in some cases, the two may overlap. Its position is between $1.5-5 \mathrm{~mm}$ from the fusion zone. This and the previous zone may be the result of total or partial solubility during the welding process with low diffusion of alloying elements, where the subsequent heat effect by thermal capacitance generates heterogenous precipitation in the second phases.

The existence of a fifth zone is detected, characterized by a slight decrease in microhardness, around $75 \%$ of base metal hardness. This occurs in what many authors consider to be the HAZ. The minimum value is observed some $15-20 \mathrm{~mm}$ from the fusion line and it is here that tensile fractures usually occur when they arise in places far from the bead. The increased hardness towards both sides of this zone is profiled gradually and only slightly accentuated, reaching base metal values around $25-30 \mathrm{~mm}$ for the testpieces here.

Differences were observed in microhardness evolution across the width of the HAZ in terms of the zone analysed (upper, central and lower zones). This may be because the plates were in horizontal position during welding. The convection process on the lower side of the sheet is limited, then hot air which tends to rise is trapped to form a stationary layer which cannot transfer heat, while on the upper side, convection is ensured.

A careful look at Fig. 7 reveals a slight tendency in bevelled testpieces to show microhardness decreases, at slightly greater distance but which never exceeds $0.3 \mathrm{~mm}$ in the third zone, $0.5 \mathrm{~mm}$ in the fourth and $2 \mathrm{~mm}$ in the fifth, which appears contradictory, as the heat input during welding was slightly lower in the case of joints with bevel. Conversely, microscopic examination shows that joints without bevel preparation showed a slightly larger HAZ, which may be an effect of heat input introduced during the welding process.

The various EDAX tests did not reveal any volatility or diffusion in alloying elements, from which it is deduced that microhardness decreases are exclusively due to solution and precipitation processes at the first four points and in a process of over-ageing in the fifth zone.

However, Malin, ${ }^{14}$ Lean et $\mathrm{al}^{12}$ and other authors refer to three preferential zones where welded aluminium testpieces fail. These occur at the bead centre, at the base-weld metal interface and in a zone within the HAZ but somewhat removed from the bead. This is corroborated by the results obtained and it may be considered that due to the closeness 
of zones 2, 3 and 4, which in the majority of cases did not exceed 2.0-2.5 mm, there is possible confusion when evaluating failure position.

Axial tests are direct indicators of the static behavior of a material. The different tests conducted show an appreciable decrease in strength of the welded joint compared to that of the base metal.

An aspect worthy of mention is, in the case of testpieces with no bevel, the apparently low elastic limit compared with those with bevel, but if rupture stress and elongation percentage are considered, it is observed that both rupture stress and plasticity are slightly higher in testpieces with bevels, thus showing greater safety.

Comparing the elastic limit of welded testpieces with the values obtained from the base metal, a slight difference between both procedures was observed: $57 \%$ for joints with no bevels and $66 \%$ for joints with bevel, but if rupture stress is considered, these values are of the order of $69 \%$ for those with no bevels and $67 \%$ for those with bevels, those with no bevels comparing favourably in this case. Conversely, it is observed that elongation is appreciably reduced in both cases, of the order of 40 and 37\%, which clearly indicates that deformation is concentrated on the weakest regions. Finally, it is evident that the majority of fractures occur between zones 2 and 3 and the remainder in the vicinity of zone 1 which, although it is the weakest zone (6\% below zones 2 and 3 ), this only occurs in the area of the bead corresponding to the second pass. Nevertheless, the relationship between rupture stress decrease and that of base metal is in direct relation to the order of magnitude of microhardness decrease.

\section{Conclusions}

During the investigation, the great usefulness was revealed of microhardness as a tool to evaluate weld junction behaviour. From the results obtained the following may be concluded:

1 Microhardness evolution is similar in the cases studied, with and without prepared bevel, despite the fact that for testpieces welded with bevel preparation, microhardness decreases were more marked, the greatest being around 3\%.

2 Five suitable sites where failure could occur were, however, observed in the two cases studied.

3 Volatility of chemical elements was not observed in the HAZ which may justify decreased microhardness in these zones. This decrease was justified by precipitation mechanisms.

4 Bead morphology of bevel welded testpieces is more symmetrical than those without bevels, although bead area, as well as distances and widths, are greater.

5 Contrary to what some authors have stated, the HAZ extends beyond 25-30 mm, as observed in microhardness scans, there being a weak zone $15-20 \mathrm{~mm}$ from the bead interface.

6 Edge preparation of the sheets studied, which were $5 \mathrm{~mm}$ thick, do not show any improvement in the final mechanical properties of the weld junction but rather show a slight decrease of 2-3\% compared to those with no preparation. To this may be added greater ease and economy of joint performance with no edge preparation.

\section{References}

1 Polmear I J: Overview Mater Trans JIM 199637 12-31. 
2 Debbouz O and Navaï F: J Mater Sci 199732 475-482.

3 Hermann R et al: Mater Sci Eng A 1996 247-255.

4 Hwang R T and Chou C P: Scr Mater 199838 215-221.

5 Hepples W et al; J Mater Sci 199227 5720-5726.

6 Guitterez L A et al: Weld Res Suppl 1996 (4) 115-121.

7 Gómez de Salazar J M et al: Rev Metal 199834 276, 280.

8 Yong-Seog K and Su II P: Aluminium 198359 123-127.

9 Mondolfo L F: 'Aluminium Alloys: Structure and Properties', Publ Butterworths, London-Boston, 1976.

10 American Society for Metals. 'Specialty handbook, Aluminium and aluminium alloys'. ASM International, 1993.

11 Alubook.http://www.alu-info.dk/Html/alulib/modul/albook40.htm, dic 1999.

12 Lean P P et al: 'Pulsed arc MIG welding of composite material AA6092/SiC/25p using unreinforced fillers (Al-5Mg)'. Composite Materials 99 109-116.

13 American Standard for Testing Materials. ASTM Standards, 1991.

14 Malin V: Weld Res Suppl 1995 305-318.

15 Hussain H M: Weld Res Suppl 1996 (7) 209-215.

16 Whitaker I R: Doctoral Thesis, University of Nottingham, 1994. 